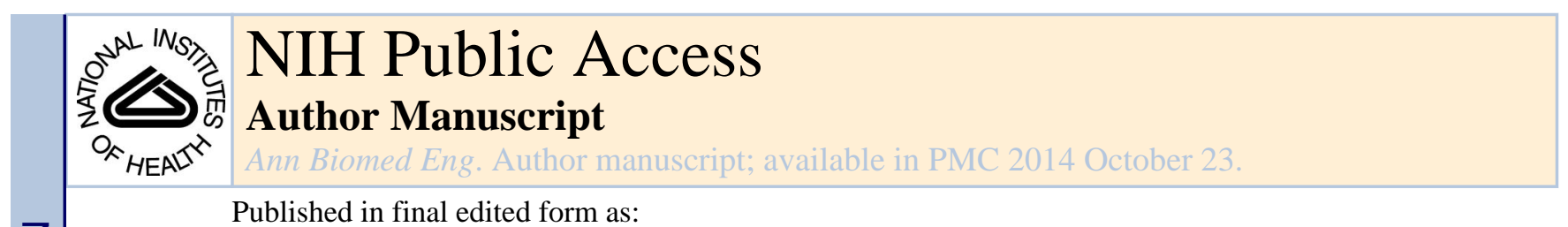

Published in final edited form as:

Ann Biomed Eng. 2011 July ; 39(7): 2010-2026. doi:10.1007/s10439-011-0285-6.

\title{
A Relation Between Near-Wall Particle-Hemodynamics and Onset of Thrombus Formation in Abdominal Aortic Aneurysms
}

\author{
C. Basciano ${ }^{1}$, C. Kleinstreuer ${ }^{2,3,4}$, S. Hyun ${ }^{5}$, and E. A. Finol 6 \\ ${ }^{1}$ Physics-Based Computing Group, Southeast Division, Applied Research Associates, Raleigh, \\ NC 27615, USA 2Department of Mechanical \& Aerospace Engineering, North Carolina State \\ University, Engineering Bldg 3, Campus Box 7910, 911 Oval Drive, Raleigh, NC 27695-7910, \\ USA ${ }^{3}$ Joint Department of Biomedical Engineering, North Carolina State University, Raleigh, NC \\ 27695, USA ${ }^{4}$ University of North Carolina at Chapel Hill, Chapel Hill, NC, USA ${ }^{5}$ Department of \\ Biomedical Engineering, Mercer University, Macon, GA 31207, USA; and ${ }^{6}$ Departments of \\ Mechanical and Biomedical Engineering, Institute for Complex Engineered Systems, Carnegie \\ Mellon University, Pittsburgh, PA 15213, USA
}

\begin{abstract}
A novel computational particle-hemodynamics analysis of key criteria for the onset of an intraluminal thrombus (ILT) in a patient-specific abdominal aortic aneurysm (AAA) is presented. The focus is on enhanced platelet and white blood cell residence times as well as their elevated surface-shear loads in near-wall regions of the AAA sac. The generalized results support the hypothesis that a patient's AAA geometry and associated particle-hemodynamics have the potential to entrap activated blood particles, which will play a role in the onset of ILT. Although the ILT history of only a single patient was considered, the modeling and simulation methodology provided allow for the development of an efficient computational tool to predict the onset of ILT formation in complex patient-specific cases.
\end{abstract}

\section{Keywords}

Patient-specific AAA; Computational analysis; ILT onset criteria

\section{INTRODUCTION}

Abdominal aortic aneurysm (AAA) rupture is the tenth leading cause of death in the United States. ${ }^{5}$ In fact, there is ample evidence that this pathological condition has the potential to be of long-term concern in the medical field. ${ }^{12}$ For this reason, it is vital to establish the physico-biochemical causes of AAA rupture ${ }^{18,42}$ and determine the best treatment options.

(C) 2011 Biomedical Engineering Society

Address correspondence to C. Kleinstreuer, Department of Mechanical \& Aerospace Engineering, North Carolina State University, Engineering Bldg 3, Campus Box 7910, 911 Oval Drive, Raleigh, NC 27695-7910, USA. ck@eos.ncsu.edu.

The research was performed in Department of Mechanical \& Aerospace Engineering, North Carolina State University, USA. 
Often associated with aneurysms is a layer of intraluminal thrombus (ILT), formed following a complex physico-biochemical process inside the aneurysm sac, which is present in approximately $75 \%$ of all AAAs. ${ }^{43}$ It has been argued that in some cases an ILT is beneficial, i.e., it forms locally a "new blood conduit" and may "dampen" the pressure-wave impact on the AAA wall. In contrast, other studies show that an ILT can be a factor in the overall risk of aneurysm rupture, potentially being a source of proteolytic activity and condensed inflammatory response as well as a site of local wall thinning, weakening, and hypoxia. ${ }^{15,38,41}$ In any case, ILT formation and growth is an ongoing process that involves a myriad of hemodynamic and biochemical stimuli, primarily including the activation, coagulation, and clotting of platelets and other blood particles. Evidence for such behavior is seen in the direct relationship between ILT and AAA volume. ${ }^{12,37,38,40}$ Hemodynamic aspects of continuous ILT development related to an expanding idealized AAA geometry were discussed by Salsac et al..$^{29,30}$ and Lasheras. ${ }^{19}$ They illustrated significant flow separations present throughout the cardiac cycle in progressively enlarging AAA geometries. Similar transient patterns of flow separation in the downstream expanding region of a stenosed vessel were shown by Raz et al. ${ }^{28}$ to entrap platelets and activate their clotting mechanism revealed by the direct relationship between recirculation time and platelet acetylated thrombin generation. Yamazumi et al. ${ }^{46}$ also showed that AAA hemodynamics exhibits an activated state of coagulation and fibrinolysis that is correlated with the aneurysm morphology (neck angle, tortuosity, and size). Contributing to the activated state of coagulation, Houard et al. ${ }^{14,15}$ reported elevated amounts of blood-borne proteases and fibrinolytic enzymes that are retained in the aneurysm lumen entrapped in the local patterns of flow recirculation. Touat et al. ${ }^{39}$ illustrated the enhanced coagulation properties of blood particles in ILT by measuring their clotting time compared against a control state of enriched media. Interestingly, the particles from the luminal ILT layer had the shortest clotting time and thus exhibited the highest degree of fibrin generation coinciding with the enhanced presence of fibrin in this ILT layer. The elevated activation level of platelets in AAAs was further illustrated by Dai et al. ${ }^{6}$ when by inhibiting platelet clotting, they found that fewer leukocytes infiltrate the ILT and the AAA wall retained a higher degree of mechanical integrity in a murine AAA model. The multi-scale modeling papers by Fogelson and Guy, ${ }^{8}$ Pivkin et al.,${ }^{26}$ and Xu et al..${ }^{45}$ are recent examples of computer simulation studies addressing fundamental processes of particle-wall interaction, platelet activation and subsequently thrombus formation.

To date there have been a few studies modeling blood particle trajectories in patient-specific AAAs. For example, Basciano et al. ${ }^{2}$ analyzed steady flow patterns influencing particle entrapment in a patient-specific AAA with elevated particle residence times and future ILT development. Salsac et al. ${ }^{31}$ used an Eulerian-Lagrangian fluid-particle methodology to model the trajectories of particles in AAAs and further confirmed that AAA hemodynamics moves platelet-like particles towards the aneurysm wall. Biasetti et al. ${ }^{3}$ analyzed the hemodynamics inside AAAs and showed elevated flow separation and recirculation when compared to the flow fields of a healthy abdominal aorta. This indicates that AAAs possess the necessary hemo-dynamic conditions coinciding with biological trends of elevated coagulation and activation of the platelet clotting mechanism. They further postulated that platelets are activated as they enter into the aneurysm bulge and, when later entrapped in the 
recirculation zones, they preferentially attach to the already existing ILT or the AAA wall in distal regions of the vessel. Their postulate is somewhat confirmed by multiple anatomic studies showing eccentric volume distributions of ILT with some prevalence seen in the distal anterior region of the AAA. ${ }^{7,11,12,23}$ Based on a computational analysis, Rayz et al. ${ }^{27}$ observed thrombus deposition in certain regions of three patient-specific geometries with an increase in "flow residence time" and low AAA wall shear stress.

Biological experiments are difficult to use for validation because they would require biopsied ILT samples, which cannot be easily obtained from an AAA patient during the course of the disease at regular clinical follow-ups. Thus, we computationally analyzed the AAA hemodynamics employing data sets obtained from a patient placed under periodic surveillance for 3 years. Of special interest were the patient-specific, biomechanical factors/ processes for the onset of ILT formation. The key ILT onset criteria considered include regionally complex hemodynamics, activation of primary cellular components of blood, mainly platelets, and blood particle entrapment in hemodynamically unique AAA regions.

\section{METHODS}

The methods for computational fluid-particle dynamics simulation of particlehemodynamics in a patient-specific AAA configuration to predict the onset of ILT formation include: construction of the computational domain, i.e., AAA geometry plus mesh generation; specification of the governing equations for blood flow and particle transport subject to appropriate inlet/outlet boundary conditions; and the hemo-dynamic criteria for ILT onset which, of course, may also relate to future ILT growth.

\section{Construction of Patient-Specific Computational Mesh}

To analyze the continual ILT formation process, a representative patient's history of AAA and ILT development was obtained with prior approval of a human subjects research protocol by the Institutional Review Board at Allegheny General Hospital (Pittsburgh, PA). The computed tomography angiography (CTA) images of the electively repaired AAA were acquired and segmented using an in-house segmentation algorithm following a procedure described by Shum et al. ${ }^{34}$ and applied by Martufi et al. ${ }^{22}$ and Shum et al. ${ }^{35,36}$ for geometric analysis and risk stratification of AAA population subsets. The 3D anatomical geometry was obtained via reconstruction of the segmented images with subsequent surface meshing, using Simpleware (Simpleware Ltd., Exeter, UK). In addition, the lumen of the geometry was truncated at the infra-renal aorta and common iliac artery locations to be normal to the centerline flow direction. CAD software Rhinoceros (McNeel North America, Seattle, WA) was used to create the normal boundary profiles.

After completion of the geometry files, ICEM CFD (ANSYS Inc., Canonsburg, PA) and SolidWorks (Dassault Syste`mes SolidWorks Corp., Concord, MA) were used to refine the normal plane at the boundaries and calculate the volumes of the AAA lumen and the ILT. Interestingly, a rapid increase in ILT volume was seen at specific times between the patient's diagnostic exams. Figure 1 depicts the patient's AAA volume history with accompanied geometric shapes (red domain is the blood lumen and tan is the ILT) at specific times, where the AAA sac volume was calculated as the sum of the AAA lumen and ILT volumes. 
The noted increase in ILT volume (see green AAA thrombus curve) is noted mainly between May 2005 and March 2006. However, while the AAA lumen volume of May 2005 may be slightly larger than the one of Decemeber 2004, that geometry has more luminal bulges and a more severe neck angle. Thus, to investigate ILT formation and test if any bulges correlate with potential particle entrapment and deposition, the December 2004 lumen geometry was used as the computational domain for the AAA particle-hemodynamics analysis.

ICEM CFD was employed to generate a mesh of the patient-specific computational AAA lumen; the lumen boundary was divided into multiple sections and prism elements of varying heights were generated along the lumen boundaries to capture accurately the high gradients of near-wall velocity flow. Variable prism heights were used to ensure they did not overtake the smaller diameters of the common iliac arteries. The distance from the no-slip boundary to the nearest node was prescribed to be $0.25 \mathrm{~mm}$ in the AAA region and $0.13 \mathrm{~mm}$ in the iliac zones, where six prism layers of equal thickness were extruded from the nearest node at the wall. The number of prism layers and their individual thickness were selected, based on trial-and-error, to accurately capture the near-wall velocity gradients for transient laminar internal flow. The other part of the flow domain was discretized with unstructured tetrahedral elements. Extensions equivalent to three times the vessel diameter of the inlet and outlet boundaries were inserted to reduce the inaccurate influence of local boundary conditions and presence of unnatural wall shear stress vectors at the AAA region proximal location. Both the inlet and outlet extensions utilized reduced extension lengths (i.e., less than five or ten times the lumen diameter as typically assumed) to achieve manageable solution/calculation times and to reduce the unnatural momentum influence the extensions would exert on the fluid streamlines and Lagrangian particles. Additionally, the circular-like cross-section and natural length of the iliac arteries enabled a reduced length for the outlet extensions to achieve stable flow through the outlets with little influence on the flow field in the AAA sac, as illustrated in Basciano. ${ }^{1}$

A mesh independence test was performed using steady flow simulations with an inlet Reynolds number of 1400, outlet boundary conditions of $100 \mathrm{mmHg}$, and a specified solver root mean square (RMS) residual of $1 \times 10^{-5}$. The previously determined prism-layer parameters were not further varied during the mesh refinement tests. The final hybrid mesh, generating velocity field and wall shear stress results independent of further refinements, had 712,304 nodes and 1,937,461 elements (1,031,125 tetrahedrals and 906,336 prisms). An important note is that the total element density is less than those reported by Les et al., ${ }^{20}$ but their domains are also larger and their geometry files have been converted to smooth surfaces (B-spline or NURBS) that are not represented by triangulated faces and allow for more extensive refinement capabilities. For the current study, the mesh refinement analysis has shown sufficient independence, yet also exceeds the spatial discretization refinement reported by many other AAA computational studies including those that reproduced experimental flow fields (e.g., Boutsianis et al. ${ }^{4}$ and Frauenfelder et al. ${ }^{10}$ ). 


\section{Transport Equations and ILT Onset Criteria}

The underlying assumptions for describing particle-hemodynamics equations include laminar flow of a blood-like fluid with one-way coupled spherical particle transport in rigid conduits. The specific flow equations and associated Quemada viscosity model for the blood rheology plus boundary conditions are given in Kleinstreuer, ${ }^{17}$ among many other sources. Thus, in light of the present topic, the focus is on the particle trajectory equations. As implied, particle-particle collisions are neglected and the particle motion does not influence the flow field of the carrier fluid. Such a model is a simplification of physical reality, but is plausible in situations where the dispersed particles form a dilute suspension, occupying a relatively small volume fraction of the local fluid elements. ${ }^{17}$ An important note is that red blood cells (RBCs) make up approximately $45 \%$ of total blood volume, which seemingly violates the use of a dilute suspension model. However, the Quemada viscosity model captures the macro-scale shear-thinning behavior of blood caused by the RBC transport and, when combined with a Lagrangian dispersed particle transport model, can provide reasonable predictions of individual $\mathrm{RBC}, \mathrm{WBC}$, and platelet trajectories through a continuum model of whole blood. ${ }^{16,17,21}$

As described by Kleinstreuer ${ }^{17}$ and documented in detail by Basciano, ${ }^{1}$ the primary forces in the governing equations needed for reasonably predicting particle trajectories in the arterial flow fields of interest are the frictional and form drag, pressure gradient, and gravitational forces:

$$
m_{\mathrm{p}} \frac{d v_{i}^{\mathrm{p}}}{d t}=F_{i}^{\mathrm{D}}+F_{i}^{\mathrm{P}}+F_{i}^{\mathrm{G}}, \quad(1 \mathrm{a})
$$

where

$$
\begin{gathered}
F_{i}^{\mathrm{D}}=\frac{1}{8} \pi \rho_{\mathrm{p}} d_{\mathrm{p}}^{2} C_{\mathrm{d}}\left(v_{i}^{\mathrm{f}}-v_{i}^{\mathrm{p}}\right)\left|v_{i}^{\mathrm{f}}-v_{i}^{\mathrm{p}}\right| \\
F_{i}^{\mathrm{P}}=-d_{\mathrm{p}}^{3} \frac{\pi}{6} \nabla_{i} p \quad(1 \mathrm{c}) \\
F_{i}^{\mathrm{G}}=\frac{\pi}{6} d_{\mathrm{p}}^{3}\left(\rho_{\mathrm{p}}-\rho_{\mathrm{p}}\right) g_{i} \quad(1 \mathrm{~d}) \\
C_{\mathrm{d}}=\frac{24}{R e_{\mathrm{p}}}\left(1+0.15 R e_{\mathrm{p}}^{0.687}\right) \quad(1 \mathrm{e}) \\
R e_{\mathrm{p}}=\frac{\rho_{\mathrm{f}}\left|v_{i}^{\mathrm{f}}-v_{i}^{\mathrm{p}}\right| d_{\mathrm{p}}}{\mu_{\mathrm{f}}} . \quad(1 \mathrm{f})
\end{gathered}
$$

Here, $F_{i}^{\mathrm{D}}, F_{i}^{\mathrm{P}}, F_{i}^{\mathrm{G}}, \mathrm{C}_{\mathrm{d}}$, and $\mathrm{Re}_{\mathrm{p}}$ are the drag force, pressure gradient force, gravity force, drag coefficient, and particle Reynolds number, respectively. The drag coefficient is 
specified with the Schiller-Naumann empirical relation that adjusts the coefficient in the transitional regions between viscous and inertial particle transport, while the particle Reynolds number is defined according to a single particle's slip velocity and diameter. The arterial wall, for particle interaction, was modeled as a perfect inelastic smooth wall, i.e., a particle deposited when touching the surface.

As postulated, particle residence time (PRT) and particle-wall shear stress (PTWSS) are two important parameters to correlate hemodynamics to ILT onset. To assist in evaluating PRT, a user-defined transport variable was created to measure the residence time distribution of the Eulerian fluid phase, which implies that a fluid element represents an equivalent "fluidparticle". Thus, the transport equation of a scalar variable $\phi$ that represents a spatially varying resident time of the Eulerian carrier fluid can be expressed as:

$$
\frac{\partial\left(\rho_{\mathrm{f}} \varphi\right)}{\partial \mathrm{t}}+\nabla_{i}\left(v_{i}^{\mathrm{f}} \rho_{\mathrm{f}} \varphi\right)=\nabla_{i}\left(\rho_{\mathrm{f}} D_{\Phi} \nabla_{i} \varphi\right)+S_{\varphi^{\prime}}
$$

where $\mathrm{D}_{\Phi}$ and $\mathrm{S}_{\phi}$ are the scalar's kinematic diffusivity and a volumetric source term, respectively. The kinematic diffusivity was set to a numerical value that would remove the term's influence on the equation's calculation since diffusive transport is negligible for the current system. Additionally, the source term was set to unity over the AAA domain and the variable's dimensions were set to seconds. As a result, the simplified transport equation reads:

$$
\frac{\partial\left(\rho_{\mathrm{f}} \varphi\right)}{\partial \mathrm{t}}+\nabla_{i}\left(v_{i}^{\mathrm{f}} \rho_{\mathrm{f}} \varphi\right)=1
$$

The time fluid "particles" require to traverse each streamline was calculated by integrating the left hand side of Eq. (2b) along the fluid streamlines.

To compute PTWSS, the average shear stress on the particles' surface was calculated from the viscous (frictional) drag of the total drag force acting on a spherical particle.

Specifically,

$$
F_{i}^{\mathrm{D}}=\iint_{S}\left(n_{j} \tau_{i j}+\nabla_{i} p \cdot l\right) d A
$$

where $n_{j} \tau_{i j}$ and $\nabla_{i} p$ are the shear stress acting on the particle surface and the pressure gradient across the object's characteristic length, respectively. After completing the surface integrals for a spherical particle, the magnitude of the total drag can be correlated with the magnitudes of the shear and pressure forces. Equations $(4 a-4 c)$ define the drag force magnitude in terms of the shear and normal force magnitudes:

$$
\begin{gathered}
\left|F_{\mathrm{D}}\right|=\left|n_{j} \bar{\tau}_{i j}\right| \cdot A_{\mathrm{s}}+\left|\nabla_{i} p\right| \cdot l \cdot A_{\mathrm{r}} \\
\left|n_{j} \bar{\tau}_{i j}\right|=\tau_{\text {eff }}^{\mathrm{P}} \quad \text { (4b) }
\end{gathered}
$$




$$
A_{\mathrm{s}}=4 \pi R_{\mathrm{p}}^{2} ; \quad A_{\mathrm{r}}=\pi R_{\mathrm{p}}^{2} ; \quad l=d_{\mathrm{p}^{\prime}}
$$

object's characteristic length, respectively. After completing the surface integrals for a spherical particle, the magnitude of the total drag can be correlated with the magnitudes of the shear and pressure forces. Equations $(4 a-4 c)$ define the drag force magnitude in terms of the shear and normal force magnitudes: where $n_{j} \bar{\tau}_{i j}, \tau_{e f f}^{\mathrm{p}} ; A_{\mathrm{s}}, A_{\mathrm{r}}, l, R_{\mathrm{p}}$, and $d_{\mathrm{p}}$ are the spatial average shear stress, the effective shear stress acting on the particle, particle surface area, particle reference area, characteristic length, particle radius, and particle diameter, respectively. The drag force can also be calculated utilizing the drag coefficient of a solid, impermeable object suspended in flow according to Eq. (5):

$$
\underline{\left|F_{\mathrm{D}}\right|}=C_{\mathrm{D}} \frac{\rho_{\mathrm{f}}}{2}\left|u^{\mathrm{r}}\right|^{2} A_{\mathrm{r}^{\prime}}
$$

where $C_{\mathrm{D}},\left|u^{\mathrm{r}}\right|$; and $\rho_{\mathrm{f}}$ are the drag coefficient, the speed of the sphere relative to the fluid speed and the fluid density, respectively. A single equation relating the effective shear stress on the particle's surface can thus be derived by substituting Eqs. (4b), (4c), and (5) into Eq. (4a), yielding:

$$
\tau_{e f f}^{\mathrm{p}}=0.25\left[C_{\mathrm{D}} \frac{\rho_{\mathrm{r}}}{2}\left|u^{\mathrm{r}}\right|^{2}-\left|\nabla_{i} p\right| \cdot d_{\mathrm{p}}\right] .
$$

The relation can be further defined according to specific particle-track variables calculated in the fluid-particle hemodynamics simulations by substituting the Schiller-Naumann drag coefficient (see Eq. 1e) and the particle slip velocity $\left(v_{\mathrm{p}}^{\mathrm{r}}\right)$ for $C_{\mathrm{D}}$ and $u$, respectively. As a result,

$$
\tau_{e f f}^{\mathrm{p}}=0.25\left[12 \frac{\rho_{\mathrm{f}}}{R e_{\mathrm{p}}} \cdot\left|v_{\mathrm{p}}^{\mathrm{r}}\right|^{2} \cdot\left(1+0.15\left(R e_{\mathrm{p}}\right)^{0.687}\right)-\left|\nabla_{i} p\right| \cdot d_{\mathrm{p}}\right]
$$

Hence, the effective shear stress acting on the cellular components of blood can be calculated to assess the potential role of platelet (PLT) and white blood cell (WBC) shear stress activation in the elevated physiological conditions of blood coagulation and clotting in AAAs, and hence ILT formation/growth.

\section{Numerical Method and Model Validations}

The commercial finite-volume software ANSYS CFX v12 (ANSYS Inc., Canonsburg, PA) was employed to solve the conservation equations of fluid and particle transport. A highresolution iterative upwind solution scheme was used to integrate the advection term in the flow equations. The high-resolution scheme uses nonlinear blending factors at each node to lessen the smearing of high spatial gradients by a first-order upwind difference scheme, while preventing dispersive discretization errors of linear blend factors and/or central difference schemes in regions of high spatial gradients. An iterative, second-order backwards Euler transient solution scheme continued until the specified mass and 
momentum maximum error residuals were less than the user-specified threshold value. Each particle's position was calculated using forward Euler integration of the particle velocity with an integration time-step $(\delta t)$ :

$$
x_{i}^{p N}=x_{i}^{\mathrm{p} 0}+v_{i}^{\mathrm{p} 0} \delta t,
$$

where $x_{i}^{p N}, x_{i}^{\mathrm{p} 0}$, and $v_{i}^{\mathrm{p} 0}$ are the new particle position at the end of the particle integration time-step $(\delta t)$ the initial particle position at the beginning of the particle integration timestep and the velocity at the beginning of the particle integration time-step, respectively. At the end of the particle integration time-step, the new particle velocity was calculated by the analytical solution to Eq. (1a). The transient, iterative solution procedure used a constant time-step of $1.25 \times 10^{-3} \mathrm{~s}$ and continued until RMS mass and momentum maximum residuals were less than $1.0 \times 10^{-5}$. The selected time-step was two orders of magnitude below the time needed for a fluid-particle traveling at the maximum inlet velocity through the domain. Twenty sub-iterations per time-step were executed, which was sufficient to achieve solution convergence. Spherical particles representing RBCs, WBCs, and PLTs were injected at the extension's inlet with zero slip velocity and a bulk mass flow rate equaling the spatially averaged carrier fluid mass flow rate at the inlet boundary. All particles were injected with a uniform, equally spaced distribution across the inlet surface, and each particle type had the same number of injected particles over time. The particle injection rate was specified by multiplying an assumed local concentration (i.e., 525 particles $/ \mathrm{cm}^{3}$ ) by the fluid's transient bulk mass flow rate. Thus, the number of injected particles varied throughout the transient inflow waveform and a total amount of approximately 10,090 particles for each particle type were injected during one period. While equal concentrations of RBC, WBC, and PLT particles is physically not realistic, the implemented concentrations enabled a large sample size of (non-interacting) PLT and WBC particles to test the ILT formation hypothesis and provided the broadest range of particle shear-loading scenarios to be included in the analysis. Particle characteristics were selected to be the following mean values:

- $\quad \mathrm{RBC}$ diameter $=8 \mu \mathrm{m} ; \mathrm{RBC}$ density $=1096.5 \mathrm{~kg} / \mathrm{m}^{3}$

- $\quad \mathrm{WBC}$ diameter $=12.5 \mu \mathrm{m} ; \mathrm{WBC}$ density $=1077.5 \mathrm{~kg} / \mathrm{m}^{3}$

- $\quad$ PLT diameter $=2 \mu \mathrm{m} ;$ PLT density $=1040 \mathrm{~kg} / \mathrm{m}^{3}$

Prior to injection of the particles, five periods of pulsatile flow were simulated in the AAA domain. Subsequently, particles were injected during the sixth period, and four additional periods were simulated to calculate the particle transport throughout the domain. Thus, ten total periods of pulsatile flow were simulated. The five periods prior to particle injection were solved in a single simulation conducted on two processors of a 64-bit Dell Precision 670 workstation with four Intel Xeon processors at $3.59 \mathrm{GHz}$ and $8 \mathrm{~GB}$ of RAM. The total simulation time was approximately $336 \mathrm{~h}$. The five periods including particle injection and transport were solved in five separate periods and were conducted on four cores of a 64-bit Dell T3500 Precision workstation with a quad core Intel Xeon E5520 processor at $2.27 \mathrm{GHz}$ and $12 \mathrm{~GB}$ of RAM. The mean simulation for each period was $36.7 \mathrm{~h}$, resulting in a total time of approximately $180 \mathrm{~h}$. Extensive computer model comparisons with benchmark 
experimental data sets were carried out by Basciano ${ }^{1}$ and some results are documented in Basciano et al. ${ }^{2}$ as well as in Kleinstreuer. ${ }^{17}$

\section{RESULTS AND DISCUSSION}

Figure 2 depicts the AAA domain with the inlet and outlet extensions, the corresponding waveforms, and the direction of the gravity vector acting on the particle trajectories. The implemented waveforms were the widely utilized flow and pressure waveforms from Olufsen $e$ e al. ${ }^{24}$ While the AAA flow data sets of Fraser et al. ${ }^{9}$ and Les et al..$^{20}$ were not directly implemented in the current study, the utilized infrarenal waveform shares many similarities with the one reported by Les et al. ${ }^{20}$ and was within the deviation bars of the waveform by Fraser et al. ${ }^{9}$ The primary difference was that the Les et al. ${ }^{20}$ infrarenal waveform has a greater amount of zero inflow present in the diastolic region of the pulse, whereas the implemented waveform has a greater amount of antegrade flow in the diastolic region of the pulse. The anticipated influence of reduced diastolic antegrade flow on particle transport in the AAA sac would be elevated particle residence and transit times, increased numbers of entrapped particles, and longer exposure times to low shear environments. Interestingly, the results of the current study would not be nullified by the aforementioned trends, but would probably be enhanced. Thus, the current study can be thought of as an attempt to model a moderate-case scenario rather than the worst-case scenario. Pressure waveforms were specified as average cross-sectional pressures at the extension outlets to allow retrograde flow in the iliac arteries and velocity vectors that are not normal to the extension's outlet cross-section.

Of special interest are the local velocity fields in the AAA sac as well as the space-time distributions of the critical blood particles (i.e., mainly platelets, PLTs, and white blood cells, WBTs), subject to varying particle shear stress loads. These results form the basis for the criteria of ILT onset/growth and comparison with the patient-specific data derived from the CTA images.

\section{Velocity Fields}

The inlet velocity fields just prior to the AAA sac region exhibited ever changing profiles with different orientations and off-center locations of the maximum velocities throughout the inflow waveform. Specifically, the profiles were often asymmetric at multiple locations and hence could not be represented by a Womersley profile, implemented without the inlet extension. The complex transient flow field, in terms of the mid-plane velocity vectors and associated color contours, is indicated in Fig. 3. An important flow characteristic is seen during the decelerating phase $(t / T=0.292)$, where the inlet flow rate is not strong enough to overcome the recirculating flow. The same flow characteristic is seen at the smaller flow rate when $t / T=0.667$ (i.e., during the diastolic phase). Moreover, at the flow reversal immediately following the decelerating inflow $(t / T=0.396)$, the local velocity in the circulation region is approximately one-half of the maximum velocity in the AAA region. Thus, recirculating flow fields are not only present for most of the cardiac cycle, but also maintain sufficient strength that will not be overcome by small inflows. 
Contours of the fluid's residence time distribution revealed an elevated increase of transit time in the AAA's distal region and unlike the steady residence time contours, localized elevated zones were present on both the anterior and posterior AAA lumen walls. Figure 4 illustrates the fluid's residence time distribution after five periods of pulsatile flow through the AAA domain. The flow recirculation in the proximal regions and shortened presence of antegrade flow in the AAA distal region are the main causes for the increase in fluid residence time. Noteworthy is that the fluid residence time predicts the lowest value for the anterior region of the AAA lumen boundary where the incoming flow is predominantly directed. While the simulation only covers $6 \mathrm{~s}$, the steady and transient residence time results support the hypothesis that the AAA causes elevated regional transit times within the sac, which may contribute to ILT formation and/or growth. The increased carrier fluid residence time can be extrapolated to suggest particles suspended in the region's flow also possess the same residence time if the particle Reynolds numbers are low, which is satisfied in the AAA sac. Moreover, the elevated wall deposition potential implied by the transient fluid residence time distributions at the AAA distal region indirectly supports the hypothesis of ILT formation postulated by Biasetti et al. ${ }^{3}$ Specifically, an increased residence would increase the potential for PLTs and WBCs, after being exposed to the circulatory flows in the proximal AAA region, to collide with the distal AAA wall.

The important secondary flows revealed that the temporal regions of maximum secondary velocity occur in the decelerating and flow reversal regions after the systolic phase. Additionally, the location of the maximum secondary velocity occurs where most of the flow reversal is present, i.e., in the proximal end of the AAA. An important deduction from the prevalent recirculation in this region of the AAA sac is the enhanced potential for shearactivated clotting mechanism of platelets. For example, Biasetti et al. ${ }^{3}$ postulated that the platelets are initially activated in the proximal regions of the AAA and then deposit, or initiate a thrombogenic response in the distal region of the AAA sac. The computed flow patterns (see Figs. 3, 5 as well as Basciano, ${ }^{1}$ for additional illustrations) support this hypothesis in that particles suspended in the proximal AAA flow would potentially traverse through several loops of the recirculation region prior to entering the distal region, which is a zone of potential stagnation due to low flow for most of the cardiac cycle. The magnitudes of time-averaged vector components may provide additional physical insight when using them as a representation of temporarily dominant conditions of pulsatile flow (see Fig. 5). Thus, they can provide a measure of re-occurring flow features of the arterial pulse. Figure 5 shows the time-averaged velocity streamlines, axial midplane velocity vectors, and secondary flow fields in the AAA. Clearly, primary flow recirculation exists in the proximal region of the AAA sac and notable elevated regions of secondary flow are caused by the neck angle directing flow towards the anterior wall.

Similar to the recirculating flow in the carotid artery for which the oscillatory shear index (OSI) was developed to indicate disturbed hemodynamics, a patient's AAA also may exhibit sustained blood recirculation as well as elevated particle residence times. To combine both,

Himburg et al. ${ }^{13}$ postulated a near-wall blood particle residence time $\left(t_{\mathrm{r}}^{O S I}\right)$ which is inversely proportional to the OSI and time-averaged wall shear stress (WSS) magnitude: 


$$
t_{\mathrm{r}}^{O S I} \sim\left[(1-2 \cdot O S I) \cdot \frac{1}{T} \int_{0}^{T}\left|\vec{\tau}_{\mathrm{w}}\right| \mathrm{d} t\right]^{-1}
$$

In the current case, the advantages of $r_{\mathrm{r}}^{O S I}$ over the fluid residence time distribution is its ability to calculate times greater than $6 \mathrm{~s}$ and having a more direct application to the suspended blood particles. Figure 6 illustrates the $r_{\mathrm{r}}^{O S I}$ contours, where the distal AAA region has localized regions of maximum residence times. An interesting observation is that locations of maximum $r_{\mathrm{r}}^{O S I}$ have a similar appearance to the particle deposition locations indicated in Fig. 12 (see also Basciano ${ }^{1}$ ). This suggests increased particle deposition in regions of elevated near-wall times. Furthermore, the maximum $r_{\mathrm{r}}^{O S I}$ contours occur at specific locations that are not always the pinnacle of local expansions of the AAA lumen.

\section{Particle Transport}

Particle transport through the transient flow field revealed that approximately $70 \%$ of all particles entering the AAA sac, exit the region (which is defined as the lumen distal to the AAA neck and proximal to the aorto-iliac bifurcation) in less than 6 s, i.e., during five continuous pulses, with about $1 \%$ of all particle types depositing on the AAA sac wall. Of the particles exiting the AAA, approximately $82 \%$ travel through the left iliac artery and $18 \%$ through the right one. The mean flow distribution has a similar trend with approximately 79 and $21 \%$ of time-averaged exiting flow traversing the left and right iliac arteries, respectively, because of the patient-specific morphologic characteristics of the iliac arteries. Of interest is the exposure and fate of all particles remaining in the AAA for a while. Specifically, individual trajectories of particles not exiting the AAA domain were processed with a custom Perl script in CFX Post v.12.1 (ANSYS Inc., Canonsburg, PA), and exported for further processing with several custom MATLAB routines (see Appendix VI in Basciano $^{1}$ ). The MATLAB routines determined particle behavior near the AAA wall and in specific regions inside the AAA sac. A near-wall region was defined as $1 \mathrm{~mm}$ from the AAA lumen's minimum circular radius extending from the axial position's local centroid (see Fig. 7). Twelve regional zones were constructed based on the axial and cross-sectional positions inside the AAA sac (see Fig. 8).

The number of particles, regional particle transit time, and particle shear stress loads were calculated in the near-wall portion of each region for each particle entering the corresponding region that remained in the AAA sac after four periods of pulsatile flow. The transient movement of particles in and out of the near-wall regions is represented by the number of particles at the end of the four periods of pulsatile flow following particle injection.

A three-dimensional visualization of the near-wall particle transport is depicted in Fig. 9, where the PLTs are shown as points throughout a wireframe of the AAA sac. This shows a clear progression of particles leaving the proximal near-wall regions and entering the middle and distal near-wall regions with increasing time. Despite the continued trend, particles were still present in the proximal AAA region at the end of the four cardiac cycles, revealing the 
potential of particles to be entrapped in the AAA domain, where they may contribute to ILT formation and growth. Spatial distributions of particles in each of the quadrants and regions exhibited some dependence on time and particle type. However, the general behavior and number of particles in each region were similar and most of the increases or decreases in regional particle presence over time were observed for all three particle types.

\section{Shear-Activated Particles}

Particles that remain in the AAA sac for extended periods of time have increased exposure to the AAA's pathologic flow conditions. ${ }^{3}$ For example, to assess the potential of shearinduced clotting and inflammatory activity of the PLTs and WBCs caused by the AAA hemodynamics, the shear stress acting on the surfaces of near-wall PLTs and WBCs was calculated with Eq. (7). Box-and-whisker plots in Fig. 10 depict the shear stress on all PLTs and WBCs remaining in the AAA sac over the final three periods of pulsatile flow. The periodic stress loading of all particles and its wide variations throughout the AAA sac are well illustrated. Each vertical collection of data represents all particle shear stresses at a single instant in time. The horizontal red line is the median of the sample, the blue box outlines the upper and lower quartiles of the sample, and the vertical bars extending from the boxes represent $1.5 \times$ the interquartile range. Red + signs represent data points that lie outside the range of the vertical bars. The sample trends of periodic shear loading illustrate a dependence on particle type and residence time. Interestingly, the diastolic region of the inflow waveform corresponds with particle shear stresses less than 200 dynes $/ \mathrm{cm}^{2}$ for PLTs and less than 40 dynes $/ \mathrm{cm}^{2}$ for WBCs. Thus, conditions of potentially elevated shear stress may be followed by lower shear. Sheriff et al. ${ }^{33}$ showed that once PLTs are activated by fluid shear stresses at or above 60 dynes $/ \mathrm{cm}^{2}$ for $40 \mathrm{~s}$, they become highly sensitive to subsequent low shear values. Shankaran et al. ${ }^{32}$ found that platelets exposed to fluid shear stresses at or above $80 \mathrm{dyne} / \mathrm{cm}^{2}$ were sufficiently activated and that platelets expressed stages of coagulation within $10 \mathrm{~s}$ of shear stress exposure. Hence, the shear loads in the total AAA sac may lead to shear-induced activation of PLTs, which would begin forming the fibrin mesh necessary for ILT formation and growth. In summary, the shear stress loads in the near-wall regions of the lumen are crucial for assessing potential activation states of PLTs and WBCs near the wall.

Due to the novel use of Eq. (7) to predict shear stress on micron-sized particles, the numerical values were compared to the spatially averaged wall shear stress obtained assuming Stokes flow around a sphere (see Panton ${ }^{25}$ ). Table 1 lists the input parameters for WBCs and PLTs, which were extreme values in the simulation results. The Stokes shear stresses yielded the values displayed in Fig. 10 within reasonable limits as set by the fluidparticle parameters.

\section{Intraluminal Thrombus Development}

The particle-hemodynamics simulations of PLTs, RBCs, and WBCs in the AAA domain revealed localized regions of enhanced fluid and particle residence times. Specifically, Fig. 11a compares the ILT volume increase (calculated using reconstructed geometries of the AAA's images over time, i.e., between December 2004 and March 2006) with averaged particle transit times. The ten highest particle transit times for PLTs and WBCs within each 
region were selected because of the higher probability of coagulation and deposition. The middle segments (i.e., region numbers 5-8), the posterior segments in the proximal region (i.e., region numbers 1 and 4), and the anterior segments in the distal region (i.e., region numbers 9 and 10) showed higher ILT volume increases over time. The corresponding particle transit times for PLTs and WBCs are statistically meaningful for the prediction of ILT formation and growth (i.e., $p$ value $<0.05$ ). This further demonstrates that the particle transit time appears to be a major indicator of the formation and growth of ILT in the AAA.

ILT volume changes for different near-wall particle transit time are shown in Fig. 11b with linear regressions. Considering all data points, 68 and $65 \%$ of the variations in ILT volume change can be explained by the variation in the near-wall particle transit times for PLT and WBC, respectively. However, when specific outliers (i.e., $2 \times 2$ circled data points) are excluded from the set, there is a drastic jump in the correlation coefficient. Indeed, $r^{2}$ values for PLT and WBC near-wall transit times are now increased to 0.83 and 0.88 , respectively.

The comparison of regions of elevated residence time, OSI, and particle deposition of the current AAA lumen with future regions of ILT development in the current lumen geometry reveal a qualitative correlation between previous particle-hemodynamics results and future

ILT development. Specifically, the localized regions of elevated $r_{\mathrm{r}}^{O S I}$ correlate strongly with particle deposition locations, all indicating regions of future ILT development (Fig. 12) as assessed by the increased ILT volume seen in the subsequent CTA reconstructions. Local particle residence times in twelve different spatial, near-wall regions revealed an anisotropic distribution of maximum particle residence times, which supports the hypothesis that particles can become entrapped in the AAA domain. Shear stress calculations were then completed and revealed that PLTs, in particular, experienced high levels of shear stress at both the near-wall and throughout the AAA lumen. Moreover, the maximum time-averaged shear stress of the particles was observed to be generally greater in the proximal and middle AAA regions when compared to the distal AAA region. Experimental studies by Sheriff $e t$ al. ${ }^{33}$ and Raz et al. ${ }^{28}$ revealed sufficient platelet activation under shear stresses of similar magnitude and extended duration. However, Wurzinger et al. ${ }^{44}$ showed that brief exposures to elevated shear stresses of even a few milliseconds can lead to platelet activation. These experiments and our computational results support the hypothesis presented by Biasetti $e t$ $a l .{ }^{3}$ that shear-induced activation of blood particles causes thrombogenic and inflammatory responses in the proximal portions of the AAA sac, and may initiate fibrogenesis upon reaching the posterior AAA region. Furthermore, the study's computational results of elevated residence times support the hypothesis that the AAA sac entraps blood particles, which plays a role in ILT formation and/or growth.

Figure 12 attempts to provide a unifying illustration of the patient's AAA geometries over time and relations between ILT formation and particle-hemodynamics. Specifically, while Fig. 1 shows the history of the patient's AAA domain, Fig. 12 presents a cut-away view of the patient's future AAA geometry with fully developed thrombus and identifies specific regions that correlate with the critical particle-hemodynamics parameters. 


\section{CONCLUSIONS}

The present research study utilized established numerical methods to calculate the fluidparticle transport of RBCS, WBCs, and PLTs through a patient-specific AAA sac. Regional particle-hemodynamic parameters that depended on patient-specific characteristics (e.g., particle residence time and point-shear loads) were calculated using customized processing codes, related to the regional onset of a patient's ILT. The semi-empirical approach to capture shear stress induced PLT and WBC activation is a limitation of the computer simulation model. In general, to the authors' knowledge, this study is the first of its kind to illustrate a relation between a patient's AAA particle-hemodynamics with patient-specific ILT formation. The modeling and simulation methodology provided allow for the development of a computational tool to predict the onset of ILT formation in complex patient-specific cases. Thus, future studies of similar scope will hopefully enable multiple patient-specific AAA particle-hemodynamic analyses and increase clinicians' ability to treat AAAs by developing a predictive tool for ILT development.

\section{Acknowledgments}

The authors would like to thank Dr. Satish Muluk and the Department of Radiology at Allegheny General Hospital for supplying the CT images used in our study, as well as Ms Julie $\mathrm{Ng}$ for assisting in the segmentation/surface meshing of the AAA models and Ms Emily Childress for assisting in the finalization of the manuscript. We also acknowledge research funding from the National Heart, Lung, and Blood Institute (R15HL087268) and Carnegie Mellon University's Biomedical Engineering Department. The content is solely the responsibility of the authors and does not necessarily represent the offcial views of the National Heart, Lung, and Blood Institute or the National Institutes of Health. The authors also acknowledge the use of ANSYS® Academic Research, Release 12.1 (ANSYS Inc., Canonsburg, PA) for all computational fluid-particle simulations.

\section{REFERENCES}

1. Basciano, CA. PhD Dissertation. North Carolina State University, Department of Mechanical and Aerospace Engineering; 2010. Computational particle-hemodynamics analysis applied to an abdominal aortic aneurysm with thrombus and microsphere-targeting of liver tumors..

2. Basciano, CA.; Ng, JHY.; Finol, EA.; Kleinstreuer, C. A relation between particle hemodynamics and intraluminal thrombus formation in abdominal aortic aneurysms.. Proceedings of the ASME Summer Bioengineering Conference; Lake Tahoe, CA, USA. June 17-21; 2009.

3. Biasetti J, Gasser TC, Auer M, Hedin U, Labruto F. Hemodynamics of the normal aorta compared to fusiform and saccular abdominal aortic aneurysms with emphasis on a potential thrombus formation mechanics. Ann. Biomed. Eng. 2010; 38(2):380-390. [PubMed: 19936925]

4. Boutsianis E, Guala M, Olgac U, Wildermuth S, Hoyer K, Ventikos Y, Poulikakos D. CFD and PTV steady flow investigation in an anatomically accurate abdominal aortic aneurysm. J. Biomech. Eng. 2009; 131:011008. [PubMed: 19045924]

5. Choke E, Cockerill G, Wilson WRW, Sayed S, Dawson J, Loftus I, Thompson MM. A review of biological factors implicated in abdominal aortic aneurysm rupture. Eur. J. Vascular Surg. 2005; 30:227-244.

6. Dai J, Louedec L, Philippe M, Michel J-B, Houard X. Effect of blocking platelet activation with AZD6140 on development of abdominal aortic aneurysm in a rat aneurysmal model. J. Vasc. Surg. 2009; 49:719-727. [PubMed: 19028049]

7. de Vega Céniga M, Gómez R, Estallo L, de la Fuente N, Viviens B, Barba A. Analysis of expansion patterns in 4-4.9 cm abdominal aortic aneurysms. Ann. Vasc. Surg. 2008; 22:37-44. [PubMed: 18083334]

8. Fogelson AL, Guy RD. Platelet-wall interactions in continuum models of platelet thrombosis: formulation and numerical solution. Math. Med. Biol. 2004; 21(4):293-334. [PubMed: 15567887] 
9. Fraser KH, Meagher S, Blake JR, Easson WJ, Hoskins PJ. Characterization of abdominal aortic velocity waveform in patients with abdominal aortic aneurysm. Ultrasound Med. Biol. 2008; 34(1): 73-80. [PubMed: 17689855]

10. Frauenfelder T, Lotfey M, Boehm T, Wildermuth S. Computational fluid dynamics: Hemodynamic changes in abdominal aortic aneurysm after stent-graft implantation. Cardiovasc. Inter. Rad. 2006; 29:613-623.

11. Guimarães TAS, Garcia GN, Dalio MB, Bredarioli M, Bezerra CAP, Moriya T. Morphological aspects of mural thrombi deposition residual lumen route in infrarenal abdominal aortic aneurisms. Acta Cir. Bras. 2008; 23(Suppl 1):151-156. [PubMed: 18516463]

12. Hans SSS, Jareunpoon O, Balasubramaniam M, Zelenock GB. Size and location of thrombus in intact and ruptured abdominal aortic aneurysms. J. Vasc. Surg. 2005; 41(4):584-588. [PubMed: 15874920]

13. Himburg HA, Grzybowski DM, Hazel AL, LaMack JA, Li X-M, Friedman MH. Spatial comparison between wall shear stress measures and porcine arterial endothelial permeability. Am. J. Physiol. 2004; 286:H1916-H1922.

14. Houard X, Leclercq A, Fontaine V, Coutard M, Martin-Ventura J-L, Ho-Tin-Noé B, Touat Z, Meilhac O, Michel J-B. Retention and activation of blood-borne proteases in the arterial wall: implications for atherothrombosis. J. Am. Coll. Cardiol. 2006; 48(9):A3-A9.

15. Houard X, Rouzet F, Touat Z, Philippe M, Dominguez M, Fontaine V, Sarda-Mantel L, Meulemans A, Le Guludec D, Meilhac O, Michell J-B. Topology of the fibrinolytic system within the mural thrombus of human abdominal aortic aneurysms. J. Pathol. 2007; 212:20-28. [PubMed: 17352452]

16. Hyun S, Kleinstreuer C, Longest PW, Chen C. Particle-hemodynamics simulations and design options for surgical reconstruction of diseased carotid artery bifurcations. J. Biomech. Eng. 2004; 126(2):188-195. [PubMed: 15179848]

17. Kleinstreuer, C. Biofluid Dynamics: Principles and Selected Applications. CRC Press, Taylor and Francis; Boca Raton, FL: 2006. p. 492

18. Kleinstreuer C, Li Z. Analysis and computer program for rupture-risk prediction of abdominal aortic aneurysms. Biomed. Eng. Online. 2006; 5:19-32. [PubMed: 16529648]

19. Lasheras JC. The biomechanics of arterial aneurysms. Annu. Rev. Fluid Mech. 2007; 39:293-319.

20. Les AS, Shadden SC, Figueroa A, Park JM, Tedesco MM, Herfkens RJ, Dalman RL, Taylor CA. Quantification of hemodynamics in abdominal aortic aneurysms during rest and exercise using magnetic resonance imaging and computational fluid dynamics. Ann. Biomed. Eng. 2010; 38(4): 1288-1313. [PubMed: 20143263]

21. Longest PW, Kleinstreuer C, Buchanan JR. Efficient computation of micro-particle dynamics including wall effects. Comput. Fluids. 2004; 33:577-601.

22. Martufi G, Di Martino ES, Amon CH, Muluk SC, Finol EA. Three-dimensional geometrical characterization of abdominal aortic aneurysms: image-based wall thickness distribution. J. Biomech. Eng. 2009; 131(6):061015. [PubMed: 19449969]

23. Muraki N. Ultrasonic studies of the abdominal aorta with special reference to hemodynamic considerations on thrombus formation in the abdominal aortic aneurysm. J. Jpn. Coll. Angiol. 1983; 23:401-413.

24. Olufsen MS, Peskin CS, Yong Kim W, Pederson EM, Nadim A, Larsen J. Numerical simulation and experimental validation of blood flow in arteries with structured-tree outflow conditions. Ann. Biomed. Eng. 2000; 28:1281-1299. [PubMed: 11212947]

25. Panton, RL. Incompressible Flow. 3rd ed.. John Wiley and Sons Inc.; Hoboken, NJ: 2005. p. 821

26. Pivkin IV, Richardson PD, Karniadakis G. Blood flow velocity effects and role of activation delay time on growth and form of platelet thrombi. PNAS. 2006; 103(46):17164-17169. [PubMed: 17085579]

27. Rayz VL, Boussel L, Ge L, Leach JR, Martin AJ, Lawton MT, McCulloch C, Saloner D. Flow residence time and regions of intraluminal thrombus deposition in intracranial aneurysms. Ann. Biomed. Eng. 2010; 38(10):3058-3069. [PubMed: 20499185] 
28. Raz S, Einav S, Alemu Y, Bluestein D. DPIV prediction of flow induced platelet activationcomparison to numerical predictions. Ann. Biomed. Eng. 2007; 35(4):493-504. [PubMed: 17286206]

29. Salsac AV, Sparks SR, Chomaz J-M, Lasheras JC. Evolution of the wall shear stresses during progressive enlargement of symmetric abdominal aortic aneurysms. J. Fluid Mech. 2006; 560:1951.

30. Salsac AV, Sparks SR, Lasheras JC. Hemodynamic changes occurring during the progressive enlargement of abdominal aortic aneurysms. Ann. Vasc. Surg. 2004; 18:14-21. [PubMed: 14712379]

31. Salsac, AV.; Tang, R.; Lasheras, JC. Influence of hemodynamics on the formation of an intraluminal thrombus in abdominal aortic aneurysms (abstract).. Int. J. Artif. Organs; XXXVI Annual ESAO Congress; Compiègne, France. 2-5 September 2009; 2009. p. 397

32. Shankaran H, Alexandridis $P$, Neelamegham S. Aspects of hydrodynamic shear regulating shearinduced platelet activation and self-association of von willebrand factor in suspension. Blood. 2003; 101:2637-2645. [PubMed: 12456504]

33. Sheriff J, Bluestein D, Girdhar G, Jesty J. High-shear stress sensitizes platelets to subsequent lowshear conditions. Ann. Biomed. Eng. 2010; 38(4):1442-1450. [PubMed: 20135353]

34. Shum J, Di Martino ES, Goldhammer A, Goldman D, Acker L, Patel G, Ng JH, Martufi G, Finol EA. Semi-automatic vessel wall detection and quantification of wall thickness in computed tomography images of human abdominal aortic aneurysms. Med. Phys. 2010; 37:638-648. [PubMed: 20229873]

35. Shum, J.; Martufi, G.; Di Martino, ES.; Washington, CB.; Grisafi, J.; Muluk, SC.; Finol, EA. Quantitative assessment of abdominal aortic aneurysm geometry.. Ann. Biomed. Eng. doi:http:// dx.doi.org/10.1007/s10439-010-0175-3

36. Shum, J.; Xu, A.; Chatnuntawech, I.; Finol, EA. A framework for the automatic generation of surface topologies for abdominal aortic aneurysm models.. Ann. Biomed. Eng. doi:http:// dx.doi.org/10.1007/s10439-010-0165-5

37. Speelman L, Schurink GWH, Bosboom MH, Buth J, Breeuwer M, van de Vosse FN, Jacobs MH. The mechanical role of thrombus on the growth rate of an abdominal aortic aneurysm. J. Vasc. Surg. 2010; 51:19-26. [PubMed: 19944551]

38. Swedenborg J, Eriksson P. The intraluminal thrombus as a source of proteolytic activity. Ann. NY Acad. Sci. 2006; 1085:133-138. [PubMed: 17182929]

39. Touat Z, Ollivier V, Dai J, Huisse MG, Rossignol P, Meilhac O, Guillin MC, Michel JB. Renewal of mural thrombus releases plasma markers and is involved in aortic abdominal aneurysm evolution. Am. J. Pathol. 2006; 163(3):1022-1030. [PubMed: 16507915]

40. Truijers M, Fillinger MF, Renema KJW, Marra SP, Oostveen LJ, Kurvers HAJM, SchultzeKool LJ, Blankensteijn JD. In vivo imaging of changes in abdominal aortic aneurysm thrombus volume during the cardiac cycle. J. Endovasc. Ther. 2009; 16:314-319. [PubMed: 19642780]

41. Vande Geest JP, Wang DHJ, Wisniewski SR, Makaroun MS, Vorp DA. Towards a noninvasive method for determination of patient specific wall strength distribution in abdominal aortic aneurysms. Ann. Biomed. Eng. 2006; 34:1098-1106. [PubMed: 16786395]

42. Vorp DA. Biomechanics of abdominal aortic aneurysm. J. Biomech. 2007; 40:1887-1902. [PubMed: 17254589]

43. Vorp DA, Lee PC, Wang DHJ, Makaroun MS, Nemoto EM, Ogawa S, Webster MW. Association of intraluminal thrombus in abdominal aortic aneurysm with local hypoxia and wall weakening. J. Vasc. Surg. 2001; 34:291-299. [PubMed: 11496282]

44. Wurzinger LJ, Opitz R, Blasberg P, Schmid-Schonbein H. Platelet and coagulation parameters following millisecond exposure to laminar shear stress. J. Thromb. Haemost. 1985; 54:381-386.

45. Xu Z, Chen N, Kamocka MM, Rosen ED, Alber M. A multiscale model of thrombus development. J. R. Soc. Interface. 2008; 5:705-722. [PubMed: 17925274]

46. Yamazumi K, Ojiro M, Okumura H, Aikou T. An activated state of blood coagulation and fibrinolysis in patients with abdominal aortic aneurysm. Am. J. Surg. 1998; 175:297-301. [PubMed: 9568655] 


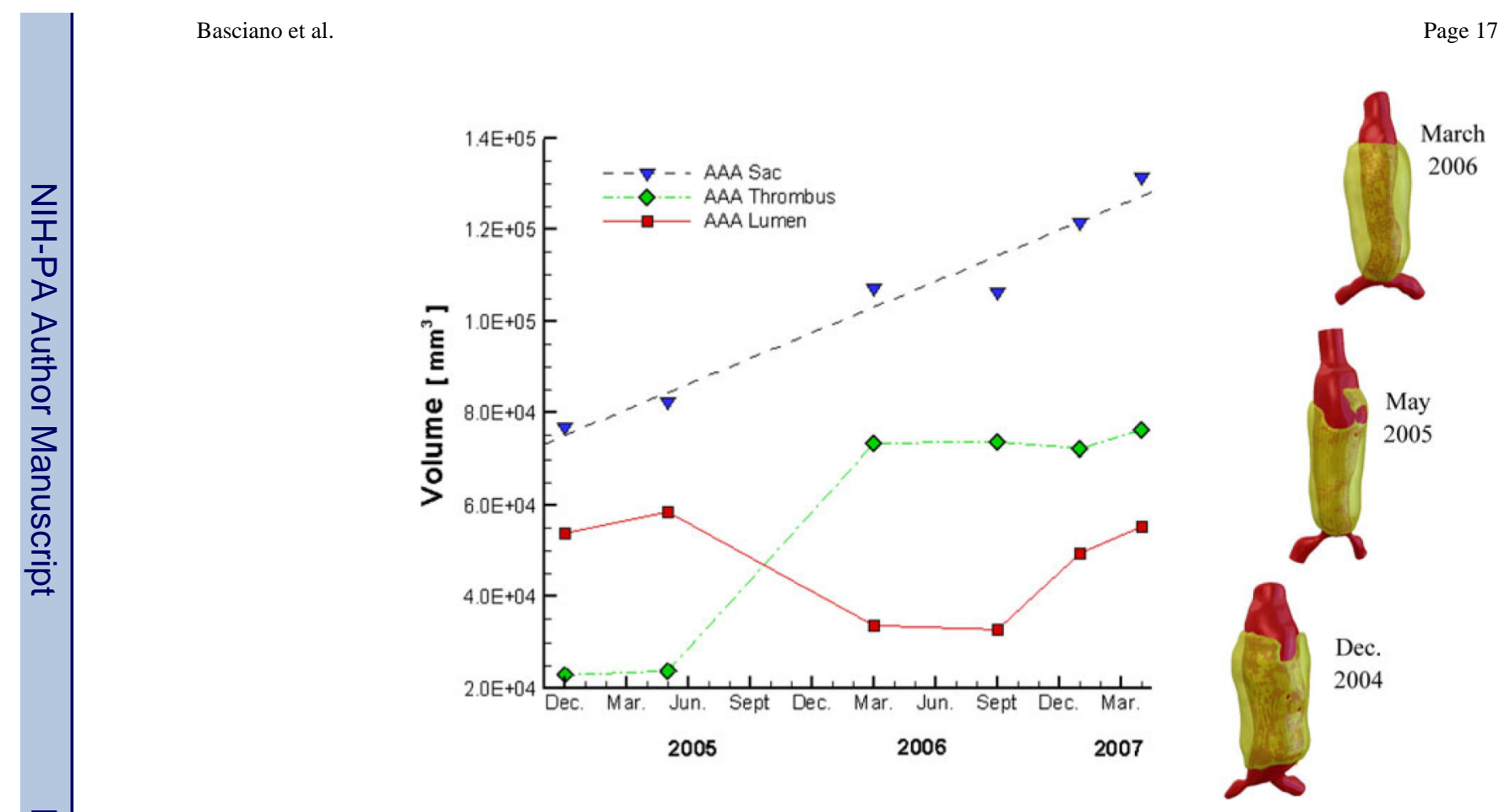

FIGURE 1.

Patient's AAA volume history and accompanying geometries (red AAA region is the blood lumen and tan AAA region is the ILT). 

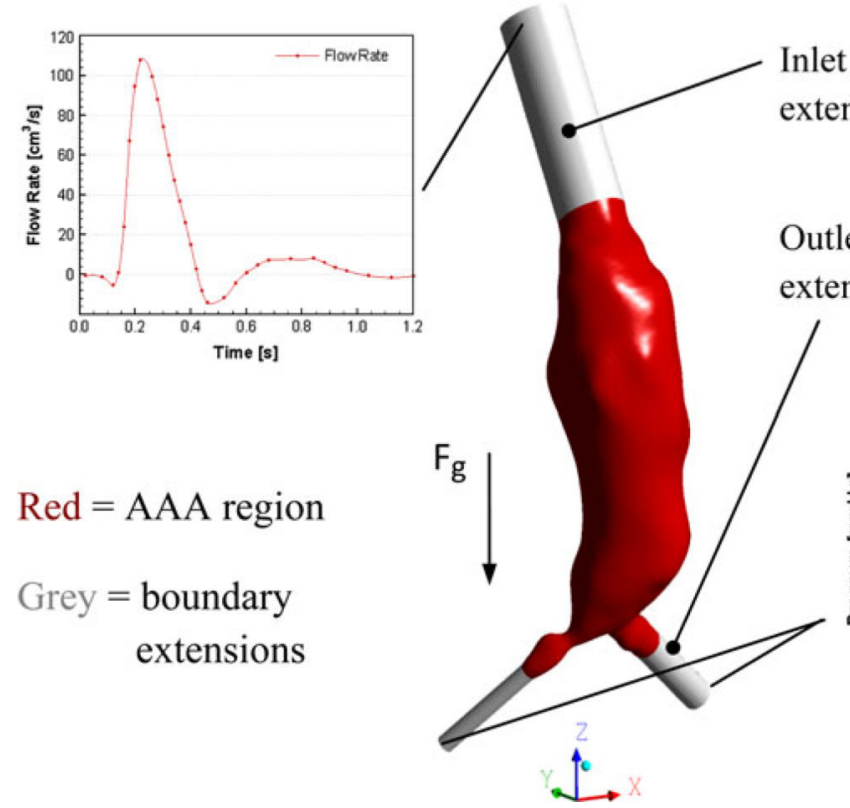
Inlet boundary extension

$$
\begin{aligned}
\text { Red }= & \text { AAA region } \\
\text { Grey }= & \text { boundary } \\
& \text { extensions }
\end{aligned}
$$

FIGURE 2.

AAA domain with inlet/outlet extensions and waveforms. 

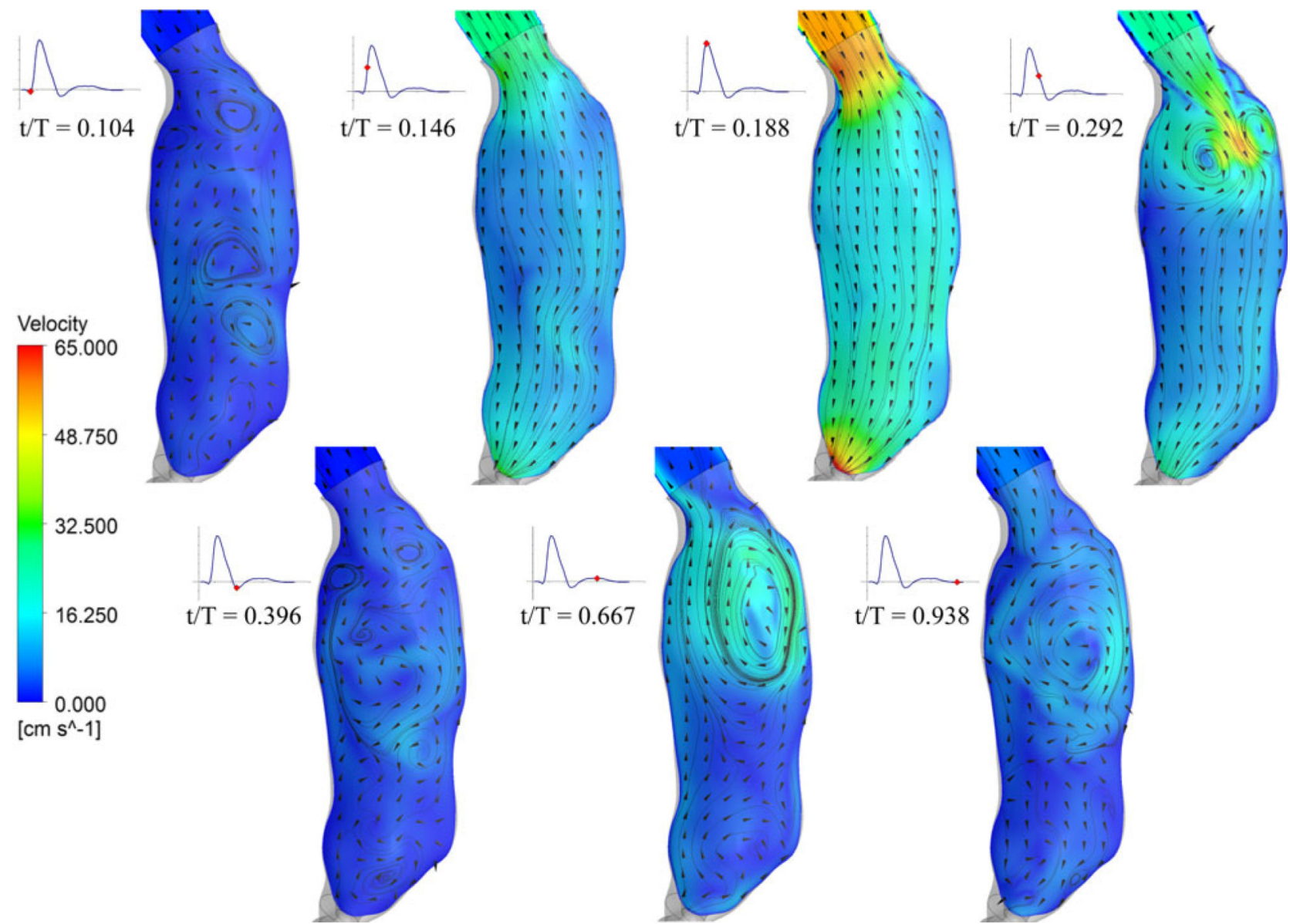

FIGURE 3.

Transient velocity vectors on axial plane of AAA sac. 


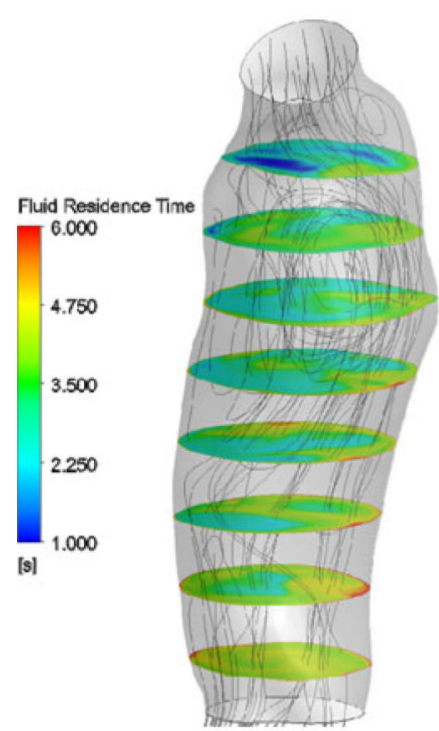

Lumen CrossSections

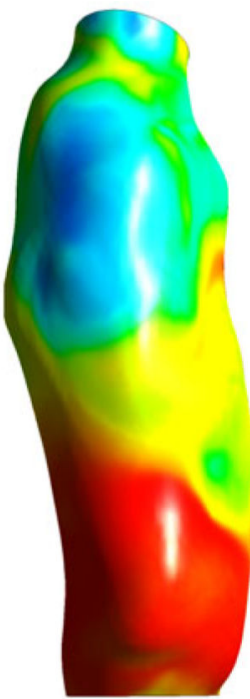

Anterior Wall

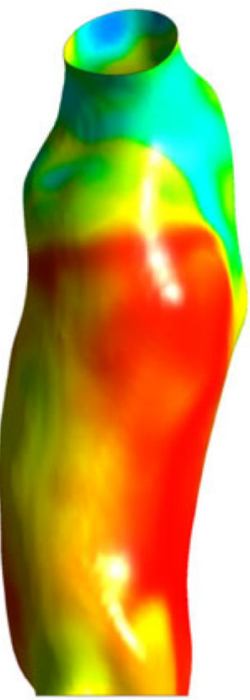

Posterior

Wall

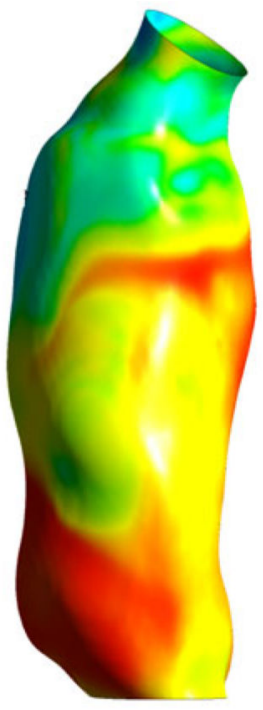

Left

Wall

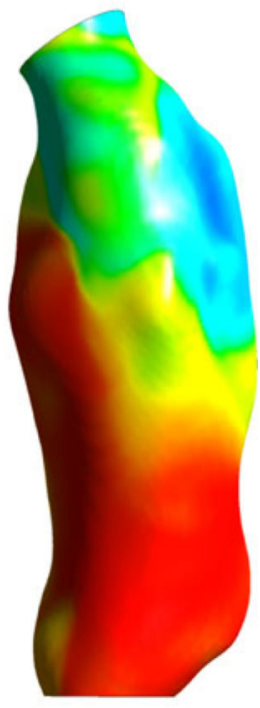

Right

Wall

FIGURE 4.

Fluid residence time distribution in AAA lumen along AAA wall after five periods of pulsatile flow. 

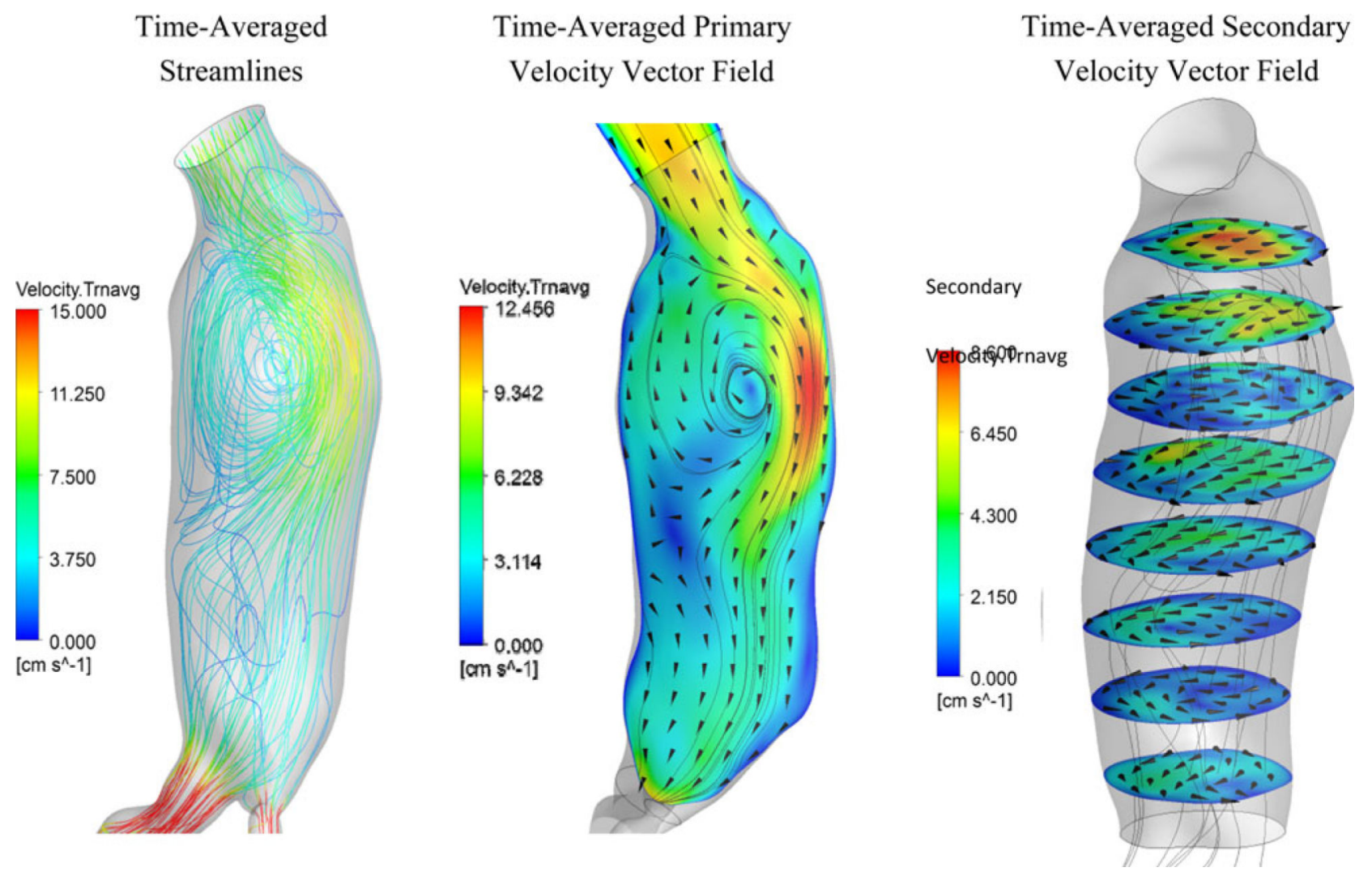

FIGURE 5.

Magnitudes of time-averaged velocity vector components. 


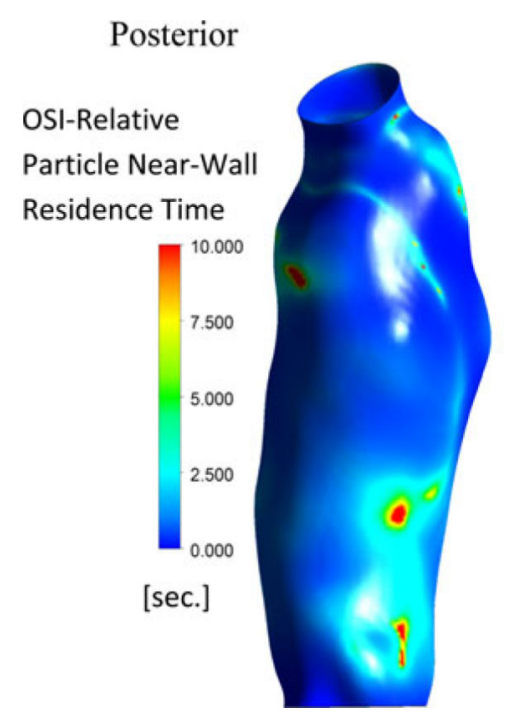

Anterior

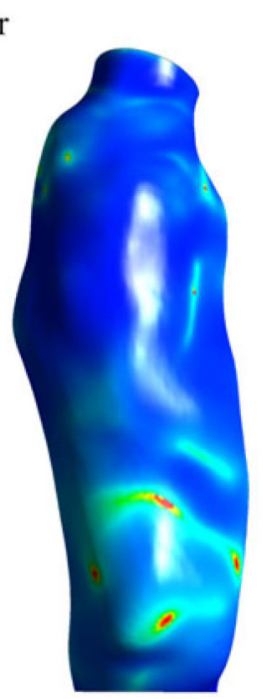

Left

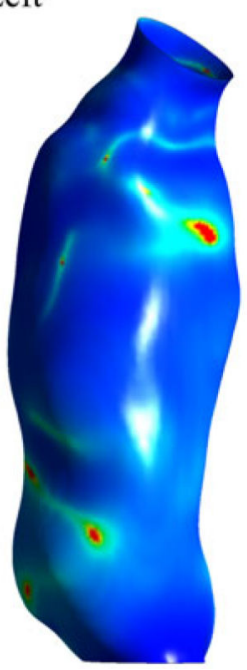

Right

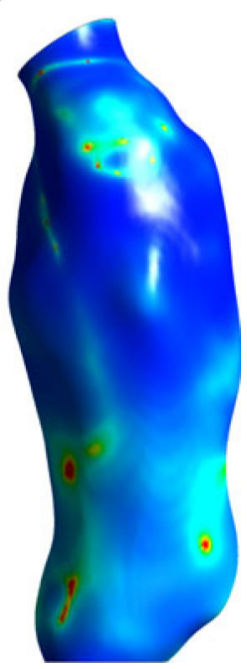

FIGURE 6.

Relative near-wall residence time derived from OSI. 


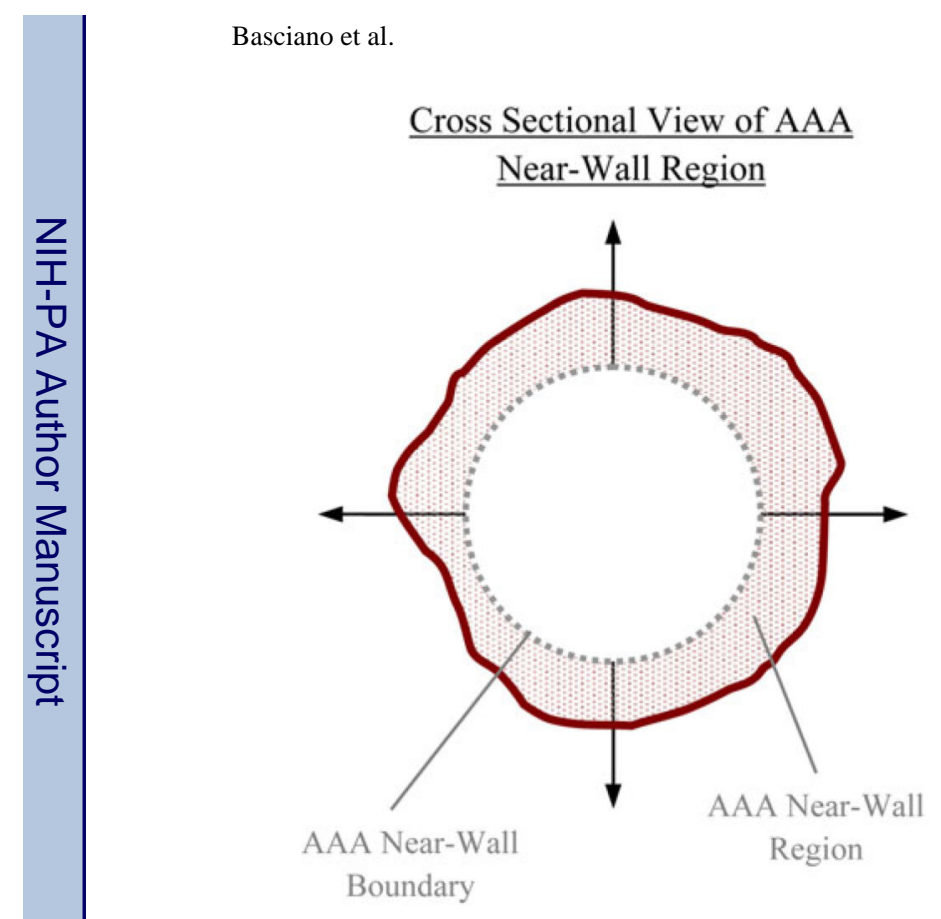

Page 23

Three-Dimensional View of AAA Near-Wall Region

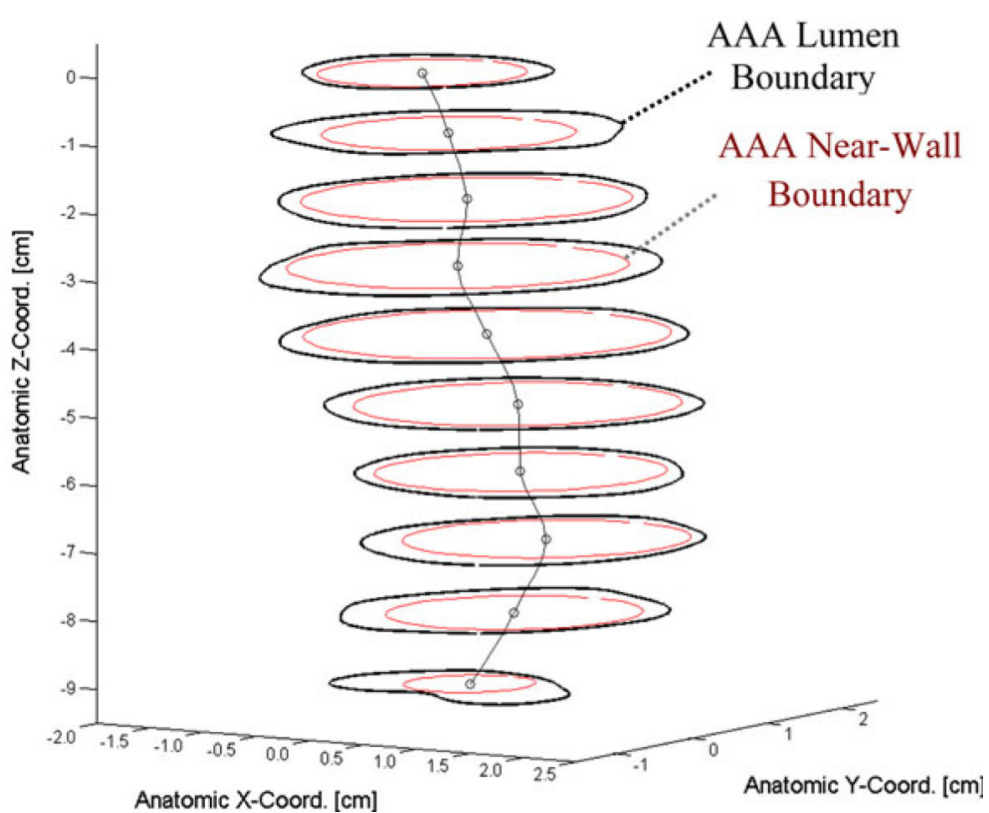

FIGURE 7.

Cross-sectional and three-dimensional views of the AAA near-wall region. 

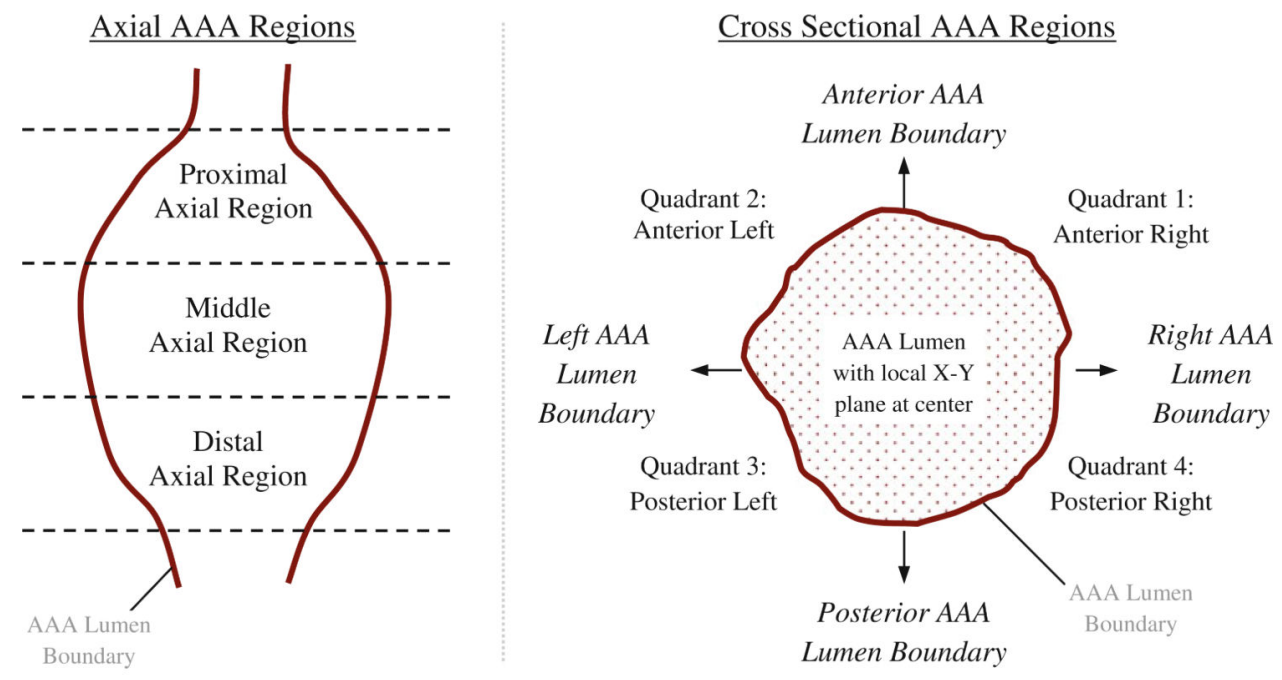

\begin{tabular}{|c|c|c|c|c|c|}
\hline$\underline{\text { Region }}$ & $\begin{array}{c}\text { Spatial } \\
\text { Description }\end{array}$ & $\underline{\underline{\text { Region }}}$ & $\begin{array}{c}\text { Number } \\
\text { Description }\end{array}$ & $\underline{\underline{\text { Region }}}$ & $\begin{array}{c}\text { Number } \\
\text { Dpatial } \\
\text { Description }\end{array}$ \\
\hline 1 & $\begin{array}{c}\text { Proximal } \\
\text { Anterior Right }\end{array}$ & 5 & $\begin{array}{c}\text { Middle Anterior } \\
\text { Right }\end{array}$ & 9 & $\begin{array}{c}\text { Distal } \\
\text { Anterior Right }\end{array}$ \\
\hline 3 & $\begin{array}{c}\text { Proximal } \\
\text { Anterior Left }\end{array}$ & 6 & $\begin{array}{c}\text { Middle Anterior } \\
\text { Left }\end{array}$ & 10 & $\begin{array}{c}\text { Distal } \\
\text { Anterior Left }\end{array}$ \\
\hline 4 & $\begin{array}{c}\text { Proximal } \\
\text { Posterior Left }\end{array}$ & 7 & $\begin{array}{c}\text { Middle Posterior } \\
\text { Left }\end{array}$ & 11 & $\begin{array}{c}\text { Distal } \\
\text { Posterior Left }\end{array}$ \\
\hline $\begin{array}{c}\text { Proximal } \\
\text { Posterior Right }\end{array}$ & 8 & $\begin{array}{c}\text { Middle Posterior } \\
\text { Right }\end{array}$ & 12 & $\begin{array}{c}\text { Distal } \\
\text { Posterior Right }\end{array}$ \\
\hline
\end{tabular}

FIGURE 8.

Spatial regions of the AAA sac with their numbering system. 

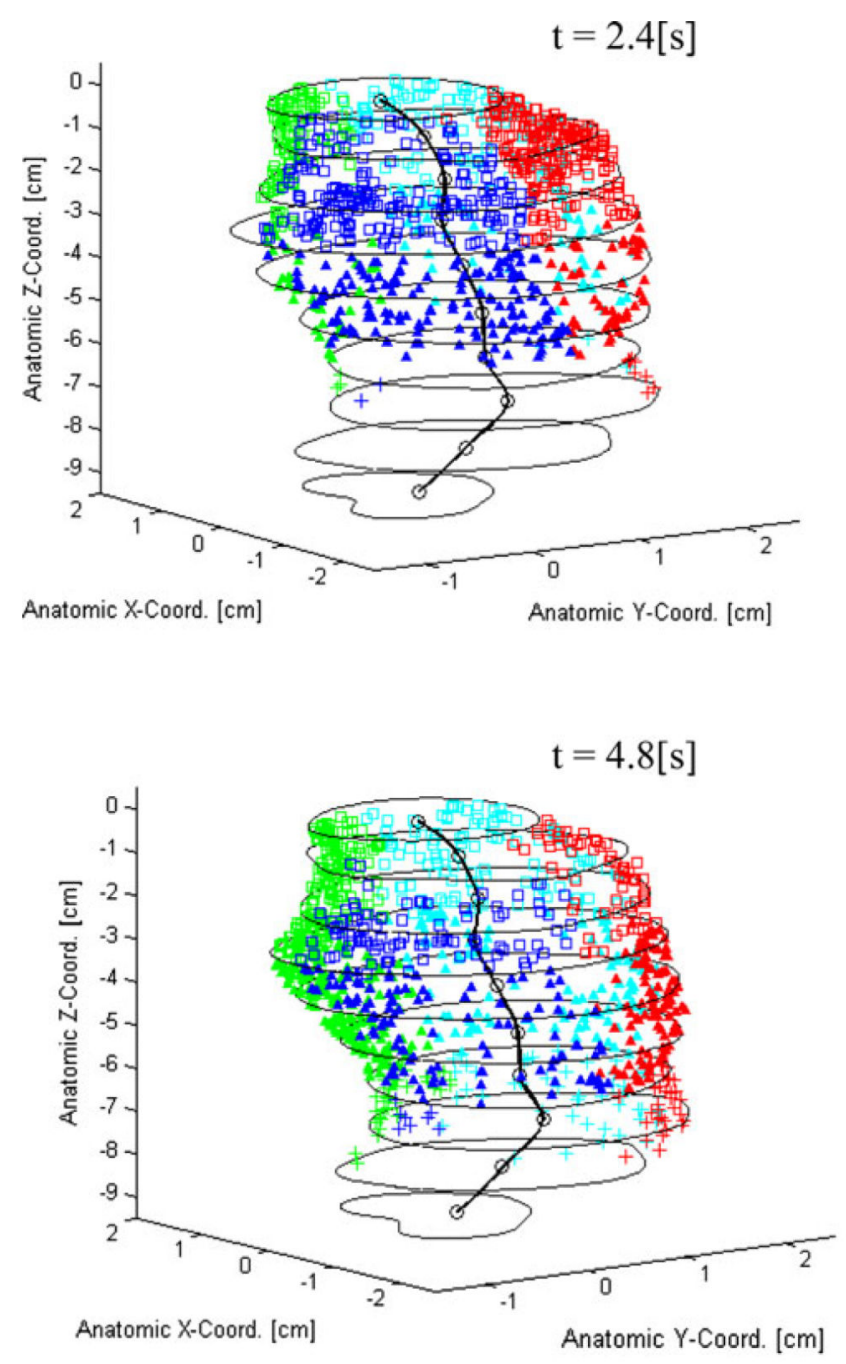

Red $=$ Quadrant 1

Cyan $=$ Quadrant 2

Green $=$ Quadrant 3

Blue $=$ Quadrant 4
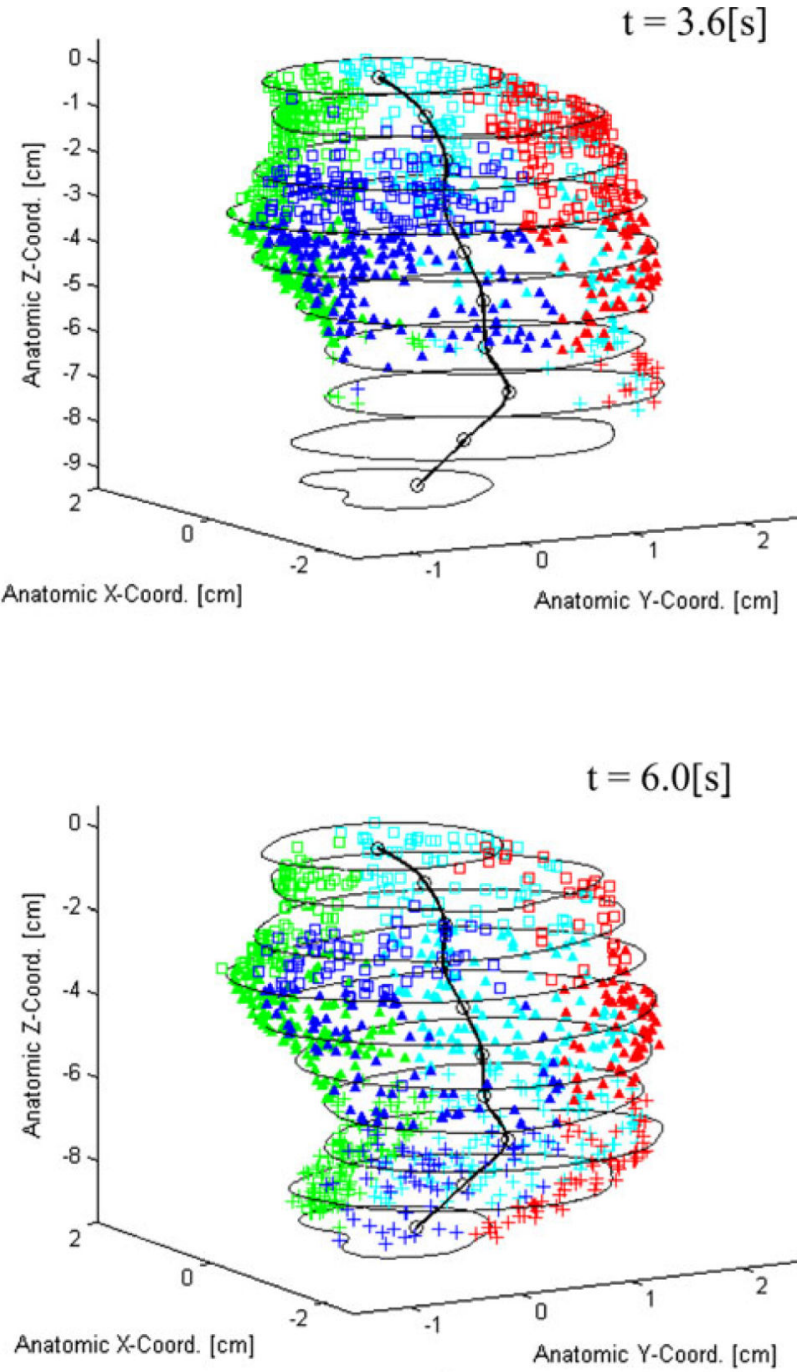

Square $(\square)=$ Proximal

Triangle $(\Delta)=$ Middle

Plus $(+)=$ Distal

FIGURE 9.

Near-wall particle distribution throughout the AAA sac at 2.4, 3.6, 4.8, and $6.0 \mathrm{~s}$. 

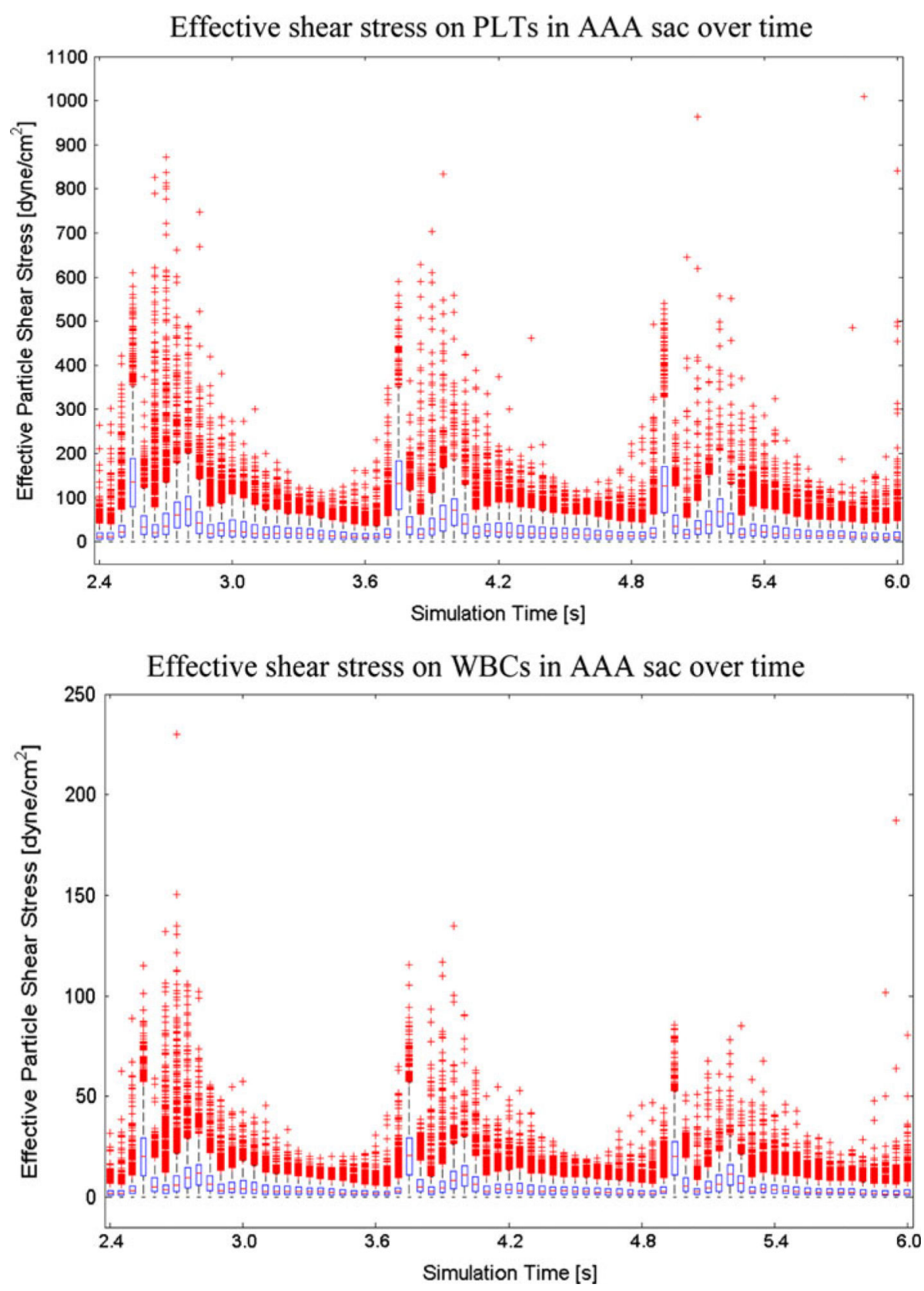

FIGURE 10.

Shear stress on the PLTs and WBCs in the AAA sac from 2.4 to $6.0 \mathrm{~s}$. 

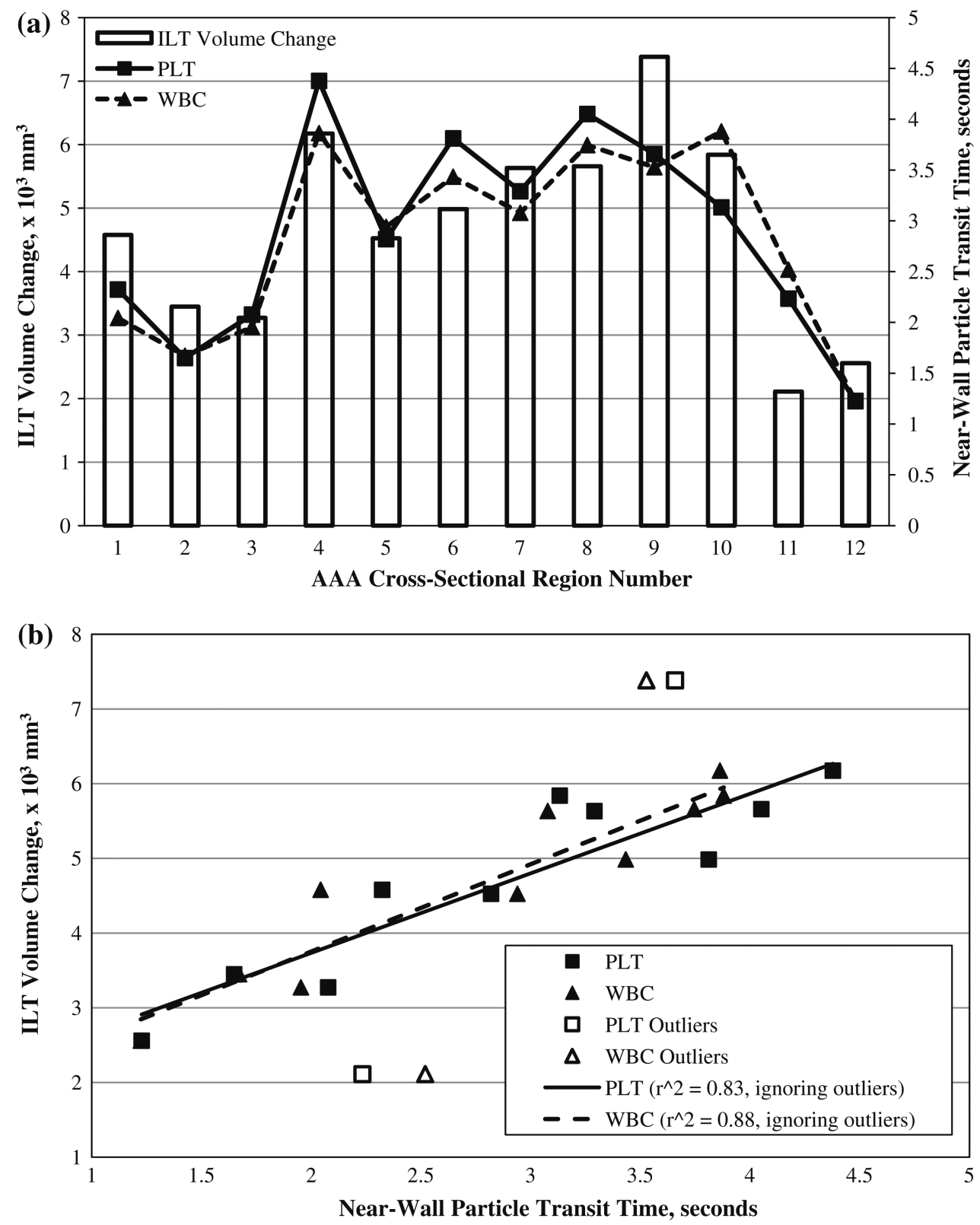

FIGURE 11.

Comparison of ILT volume change with near-wall particle transit time: (a) ILT volume change and near-wall particle transit time for each AAA cross-sectional region and (b) ILT volume changes for different near-wall particle transit time. 


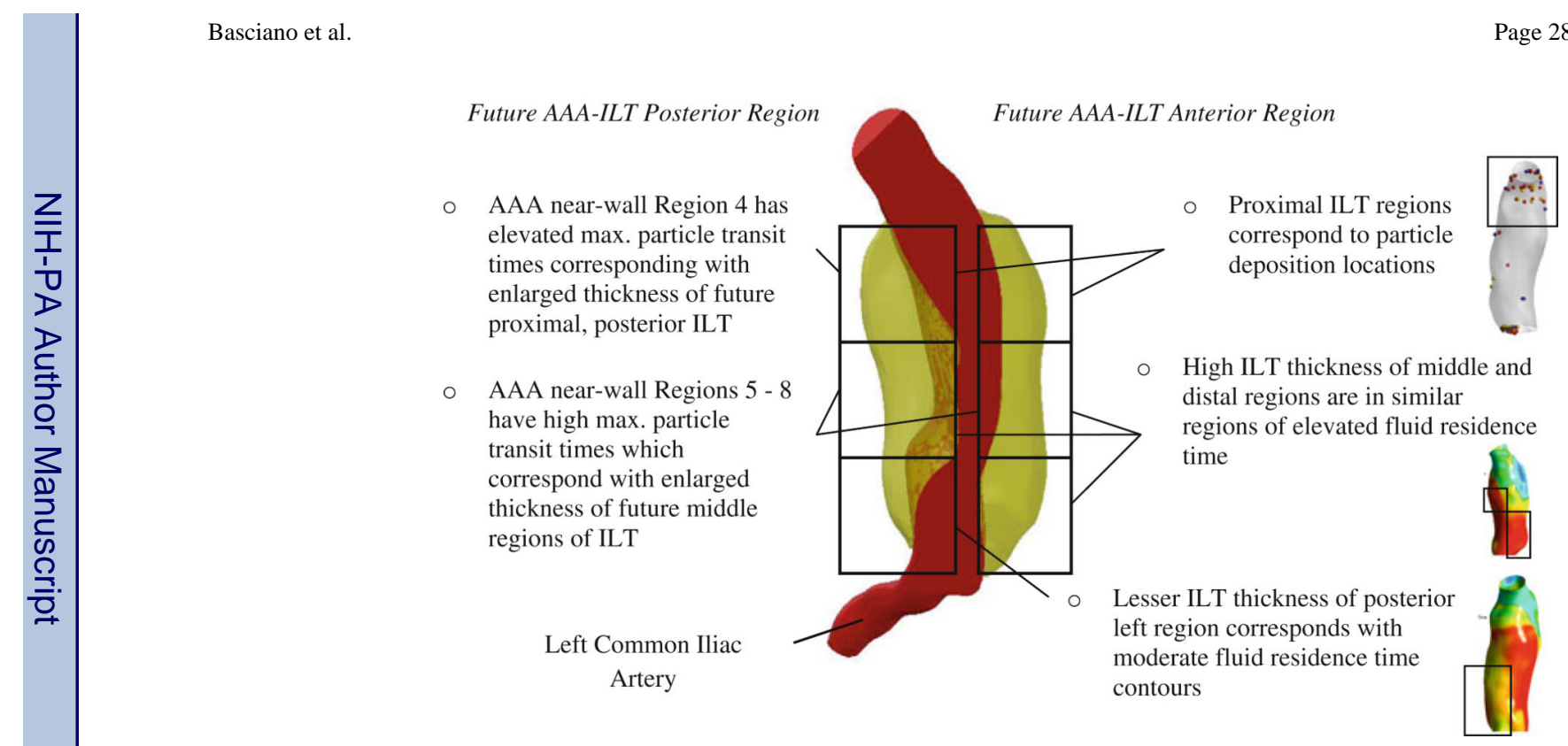

FIGURE 12.

Qualitative relation between AAA sac particle-hemodynamics and future ILT development and/or growth. 
TABLE 1

Particle shear stress calculations for Stokes flow.

\begin{tabular}{lllll}
\hline Particle & Slip velocity range $(\mathbf{c m} / \mathbf{s})$ & Particle radius $(\mathbf{c m})$ & Effective viscosity range $(\mathbf{g} / \mathbf{c m} / \mathbf{s})$ & Stokes flow shear stress $\left(\mathbf{d y n e}_{\mathbf{c m}} \mathbf{c m}^{2}\right.$ \\
\hline PLT & $1 \times 1 \mathrm{C}^{-6}-7.00$ & $2 \times 10^{-4}$ & $0.0309-0.1600$ & $1 \times 10^{-4}-6600$ \\
WBC & $1 \times 10^{-5}-4.10$ & $12.5 \times 10^{-4}$ & $0.0309-0.1600$ & $2 \times 10^{-4}-620$ \\
\hline
\end{tabular}

